\title{
Symmetry characterization and measurement errors of elasticity tensors
}

\author{
Andrej Bóna ${ }^{1}$
}

\begin{abstract}
It is often desirable to approximate a full anisotropic tensor, given by 21 independent parameters, by one with a higher symmetry. If one considers measurement errors of an elasticity tensor, the standard approaches of finding the best approximation by a higher symmetric tensor do not produce the most likely tensor. To find such a tensor, I replace the distance metric used in previous studies with one based on probability distribution functions of the errors of the measured quantities. In the case of normally distributed errors, the most likely tensor with higher symmetries coincides with the closest higher symmetric tensor, using a deviation-scaled Euclidean metric.
\end{abstract}

\section{INTRODUCTION}

With increasing accuracy of measurements in elastic materials, anisotropy of elastic materials plays an ever-important role in analyzing recorded data. However, it is usually unjustified or impractical to work with the full anisotropic stiffness tensors, given by 21 independent parameters. To this end, it is desirable to approximate the full anisotropic tensor with a tensor that possesses a higher symmetry. Only seven higher symmetries are possible, as shown by Forte and Vianello (1996), Chadwick et al. (2001), Ting (2003), and Bóna et al. (2004).

The problem of finding the symmetry class and orientation of the symmetry axes of an elasticity tensor is addressed in many papers. The exact symmetry class and its natural orientation can be found by studying its eigensystem, as shown by Bóna et al. (2007). However, in real life, the elasticity tensors contain errors and thus are almost certainly generally anisotropic. Hence, one is interested in the best approximation of the given tensor by a tensor of given symmetry.

Perhaps the first attempt to solve this problem is by Gazis et al. (1963), who set the best higher symmetric tensor to be the closest symmetric tensor using the Euclidean norm. Fedorov (1968) approaches this problem by looking at the mean square of the difference of the slowness surfaces, and Norris (2006) shows that these two approaches are equivalent. Notable among those who study the problem of approximating an elastic tensor by a tensor of higher symmetry are Arts et al. (1991) and Arts (1993). Minimizing the Euclidean distance is also equivalent to operating on the given elasticity tensor with the elements of the transformation group of the symmetry in question, as shown by Forte and Vianello (1996). Dellinger (2005) has published a numerical algorithm to find the closest tensor of higher symmetry using the Euclidean norm.

There are other ways of measuring the distance between tensors, thus providing a measure of approximation. The Riemannian distance proposed by Moakher (2006) and the log-Euclidean distance proposed by Arsigny et al. (2005) have the advantage of producing the same distance for the stiffness and compliance tensors. Moakher and Norris (2006) detail all of these distance functions.

A standard distance in the space of elasticity tensors is an abstract entity that is not related to the measurement errors. By considering a modified metric, we associate the measurement errors with this distance. In particular, we define the best approximation of an elasticity tensor by finding the most likely tensor from the higher symmetry class. The likeliness measure is given by the probability distribution that follows from considering the measurements errors.

This paper is not concerned about the way one obtains the elasticity tensor and the corresponding probability distribution. The discussion of the measurements of elasticity tensors is a separate research topic.

To illustrate why the Euclidean distance is not always the best measure of approximation of elasticity tensors, let us examine the

Manuscript received by the Editor 14 November 2008; revised manuscript received 4 March 2009; published online 28 September 2009.

Curtin University of Technology, Department of Exploration Geophysics, Perth, Australia. E-mail: a.bona @ curtin.edu.au. (c) 2009 Society of Exploration Geophysicists. All rights reserved. 
following density-scaled stiffness tensor

$$
\mathbf{c}^{0}=\left(\begin{array}{cccccc}
4 & 2.06 & 2.1 & -0.07 & 0.01 & -0.03 \\
2.06 & 3.83 & 1.96 & 0.17 & -0.07 & 0.18 \\
2.1 & 1.96 & 3.96 & 0.16 & 0.04 & -0.13 \\
-0.07 & 0.17 & 0.16 & 2 & 0.22 & -0.14 \\
0.01 & -0.07 & 0.04 & 0.22 & 1.76 & 0.02 \\
-0.03 & 0.18 & -0.13 & -0.14 & 0.02 & 2.2
\end{array}\right) \mathrm{km}^{2} / \mathrm{s}^{2}
$$

along with the corresponding measurement errors:

$$
\pm\left(\begin{array}{llllll}
1.053 & 0.897 & 0.897 & 0.39 & 0.39 & 0.39 \\
0.897 & 1.014 & 0.858 & 0.351 & 0.39 & 0.312 \\
0.897 & 0.858 & 1.053 & 0.351 & 0.39 & 0.351 \\
0.39 & 0.351 & 0.351 & 0.234 & 0.234 & 0.156 \\
0.39 & 0.39 & 0.39 & 0.234 & 0.273 & 0.195 \\
0.39 & 0.312 & 0.351 & 0.156 & 0.195 & 0.234
\end{array}\right) \mathrm{km}^{2} / \mathrm{s}^{2}
$$

If we consider the Euclidean metric, then the closest tetragonal tensor to this tensor does not fit within the measurement errors, whereas the closest transversely isotropic (TI) and cubic tensors do fit within these errors. Thus, because any TI or cubic tensor is also tetragonal, the approach of finding the closest tensor does not necessarily find the best fit within the errors. This fact is illustrated in Figure 1.

Looking for the best approximation within the error boxes would address this limitation of the shortest distance. However, we usually do not work with error boxes; we usually work with smooth error distributions. The next section discusses the use of distributions for finding the best approximation of elasticity tensor by a higher symmetric tensor.

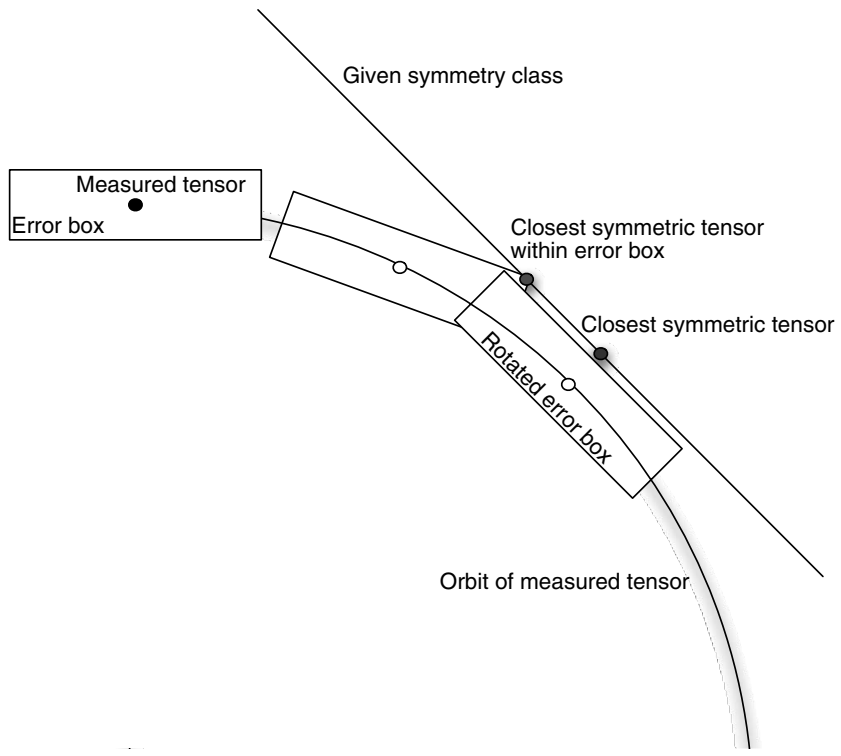

Figure 1. The closest tensor of higher symmetry to the measured tensor does not necessarily fit within the measured errors.

\section{THEORY}

Measurements of an elasticity tensor result in a probability density of the tensor. Let us denote such a density by $f(\mathbf{c})$. The form of $f$ depends on the way we measure the tensor. In the example in the Introduction, the density is given by a multidimensional boxcar function. In the example below, $f$ is given by a multidimensional Gaussian distribution.

We want to look for the most likely elasticity tensor belonging to a given symmetry class. If we denote by $S$ the linear space of tensors in the natural coordinate system of the given symmetry, we have to find the maximum of $f$ restricted to the following space:

$$
\left\{A_{i m} A_{j n} A_{k o} A_{l p} d_{\text {mnop }} \mid \mathbf{d} \in S, \mathbf{A} \in S O(3)\right\},
$$

where $S O(3)$ denotes all rotations in $\mathrm{R}^{3}$. For convenience, we work with the elasticity tensors expressed as symmetric $6 \times 6$ matrices $c_{\alpha \beta}$, where

$$
c_{\alpha \beta}=\left(\begin{array}{cccccc}
c_{1111} & c_{1122} & c_{1133} & \sqrt{2} c_{1123} & \sqrt{2} c_{1113} & \sqrt{2} c_{1112} \\
& c_{2222} & c_{2233} & \sqrt{2} c_{2223} & \sqrt{2} c_{2213} & \sqrt{2} c_{2212} \\
& & c_{3333} & \sqrt{2} c_{3323} & \sqrt{2} c_{3313} & \sqrt{2} c_{3312} \\
& & & 2 c_{2323} & 2 c_{2313} & 2 c_{2312} \\
S & Y & M & & 2 c_{1313} & 2 c_{1312} \\
& & & & & 2 c_{1212}
\end{array}\right)_{\alpha \beta},
$$

as used by, for example, Mehrabadi and Cowin (1990). The tensors in this notation rotate according the following rule:

$$
(\mathbf{A c})_{\alpha \beta}=A_{\alpha \gamma} A_{\beta \delta} c_{\gamma \delta}
$$

where $A_{\alpha \gamma} \in S O(6)$ is related to $A_{i j} \in S O(3)$ by the matrix

$$
\left(\begin{array}{cccccc}
A_{11} A_{11} & A_{12} A_{12} & A_{13} A_{13} & \sqrt{2 A_{12} A_{13}} & \sqrt{2 A_{11} A_{13}} & \sqrt{2 A_{11} A_{12}} \\
A_{21} A_{21} & A_{22} A_{22} & A_{23} A_{23} & \sqrt{2 A_{22} A_{23}} & \sqrt{2 A_{21} A_{23}} & \sqrt{2 A_{21} A_{22}} \\
A_{31} A_{31} & A_{32} A_{32} & A_{33} A_{33} & \sqrt{2} A_{32} A_{33} & \sqrt{2 A_{31} A_{33}} & \sqrt{2 A_{31} A_{32}} \\
\sqrt{2 A_{21} A_{31}} & \sqrt{2 A_{22} A_{32}} & \sqrt{2 A_{23} A_{33}} & A_{22} A_{33}+A_{23} A_{32} & A_{21} A_{33}+A_{23} A_{31} & A_{21} A_{32}+A_{22} A_{31} \\
\sqrt{2 A_{11} A_{31}} & \sqrt{2 A_{12} A_{32}} & \sqrt{2 A_{13} A_{33}} & A_{12} A_{33}+A_{13} A_{32} & A_{11} A_{33}+A_{13} A_{31} & A_{11} A_{32}+A_{12} A_{31} \\
\sqrt{2 A_{11} A_{21}} & \sqrt{2 A_{12} A_{22}} & \sqrt{2 A_{13} A_{23}} & A_{12} A_{23}+A_{13} A_{22} & A_{11} A_{23}+A_{13} A_{21} & A_{11} A_{22}+A_{12} A_{21}
\end{array}\right)
$$

Function $f$ is a function of the 21 elasticity parameters and the rotations. To simplify the description of rotations, I use the quaternion parameterization of rotations, as introduced by Hamilton (1844). More modern treatment of quaternions can be found in Altmann (1986). This representation of rotations also eliminates singularities of other representations, such as the representation by the Euler angles. We can write any rotation $\mathbf{A} \in S O(3)$ with the help of a quaternion $q=q_{0}+q_{1} i+q_{2} j+q_{3} k$ with the unit length $q_{0}^{2}+q_{1}^{2}+q_{2}^{2}+q_{3}^{2}$ $=1$ as 
$\mathbf{A}(q)$

$$
=\left(\begin{array}{lll}
1-2\left(q_{2}^{2}+q_{3}^{2}\right) & 2\left(q_{1} q_{2}-q_{0} q_{3}\right) & 2\left(q_{1} q_{3}+q_{0} q_{2}\right) \\
2\left(q_{1} q_{2}+q_{0} q_{3}\right) & 1-2\left(q_{1}^{2}+q_{3}^{2}\right) & 2\left(q_{2} q_{3}-q_{0} q_{1}\right) \\
2\left(q_{1} q_{3}-q_{0} q_{2}\right) & 2\left(q_{2} q_{3}+q_{0} q_{1}\right) & 1-2\left(q_{1}^{2}+q_{2}^{2}\right)
\end{array}\right) .
$$

This represents the rotation around the vector $\left(q_{1}, q_{2}, q_{3}\right)$ by the angle $2 \arccos q_{0}$.

In this parameterization, we can think of $f$ as a function of the 21 elasticity parameters and the four components of a quaternion. We want to find the maximum of the function $f$ subject to the following constraints. The first 21 arguments of the function are restricted to the symmetry space $S$, and the last four arguments are restricted to the four-sphere $q_{0}^{2}+q_{1}^{2}+q_{2}^{2}+q_{3}^{2}=1$. Thus, we want to find maximum of $f(\mathbf{A}(q) \mathbf{d})$, where $d \in S$ and $q_{0}^{2}+q_{1}^{2}+q_{2}^{2}+q_{3}^{2}=1$. Because $S$ is a linear space, we consider $f$ to be a function on $S$ and the space of quaternions.

The quaternions are restricted by the condition of unit length, so we formulate the problem using the Lagrange multipliers as

$$
\begin{gathered}
\frac{\partial f(\mathbf{A}(q) \mathbf{d})}{\partial d}=0, \\
\frac{\partial f(\mathbf{A}(q) \mathbf{d})}{\partial q_{i}}=2 \lambda q_{i},
\end{gathered}
$$

where $\lambda$ is the Lagrange multiplier. The derivative of $f$ with respect to a component of the quaternion $q_{i}$ is

$$
\begin{aligned}
\frac{\partial f(\mathbf{A}(q) \mathbf{d})}{\partial q_{i}} & =\left.\frac{\partial f(\mathbf{c})}{\partial c_{\gamma \delta}}\right|_{\mathbf{c}=\mathbf{A d}} \frac{\partial A_{\gamma \alpha} A_{\delta \beta} d_{\alpha \beta}}{\partial A_{\mu \nu}} \frac{\partial A_{\mu \nu}}{\partial q_{i}} \\
& =\left.\frac{\partial f(\mathbf{c})}{\partial c_{\gamma \delta}}\right|_{\mathbf{c}=\mathbf{A d}}\left(\delta_{\gamma \mu} A_{\delta \alpha}+A_{\gamma \alpha} \delta_{\delta \mu}\right) d_{\alpha \nu} \frac{\partial A_{\mu \nu}}{\partial q_{i}}
\end{aligned}
$$

and the derivative with respect to a component of the symmetric elasticity tensor $d_{\alpha \beta}$ is

$$
\begin{aligned}
\frac{\partial f(\mathbf{A}(q) \mathbf{d})}{\partial d_{\alpha \beta}} & =\left.\frac{\partial f(\mathbf{c})}{\partial c_{\gamma \delta}}\right|_{\mathbf{c}=\mathbf{A d}} \frac{\partial(A(q) d)_{\gamma \delta}}{\partial d_{\alpha \beta}} \\
& =\left.\frac{\partial f(\mathbf{c})}{\partial c_{\gamma \delta}}\right|_{\mathbf{c}=\mathbf{A d}} A_{\gamma \alpha} A_{\delta \beta} .
\end{aligned}
$$

Thus, to find the most likely higher symmetric tensor, we must solve

$$
\left.\frac{\partial f(\mathbf{c})}{\partial c_{\gamma \delta}}\right|_{\mathbf{c}=\mathbf{A d}} A_{\gamma \alpha} A_{\delta \beta}=0
$$

and

$$
\left.\frac{\partial f(\mathbf{c})}{\partial c_{\gamma \delta}}\right|_{\mathbf{c}=\mathbf{A d}}\left(\delta_{\gamma \mu} A_{\delta \alpha}+A_{\gamma \alpha} \delta_{\delta \mu}\right) d_{\alpha \nu} \frac{\partial A_{\mu \nu}}{\partial q_{i}}=2 \lambda q_{i},
$$

where $\mathbf{A}$ is given by expression 7 and $q_{0}^{2}+q_{1}^{2}+q_{2}^{2}+q_{3}^{2}=1$. The explicit form of these expressions depends on the error distribution.

\section{NORMAL DISTRIBUTION}

Let's assume that the errors in individual elasticity coefficients are independent and normally distributed. The probability density of the density-scaled elasticity tensor has the mean $\mathbf{c}^{0}$ with the standard deviations given by matrix 2 and is given by function

$$
f_{\mathbf{c}^{0}}(\mathbf{c})=\frac{\exp \left(-\frac{1}{2} \sum_{i j k l}\left(c_{i j k l}-c_{i j k l}^{0}\right)^{2} \frac{1}{\sigma_{i j k l}^{2}}\right)}{\sqrt{(2 \pi)^{21}} \prod_{i j k l} \sigma_{i j k l}},
$$

where the density function is in the tensorial notation and the indices $i, j, k, l$ run through only one of the 21 independent combinations. The form of function $f_{\mathrm{c}^{0}}$ implies that its maximum corresponds to the maximum of the argument of the exponential. Thus, in the case of normally distributed errors, the maximum probability coincides with minimizing the distance from the symmetry class, where the distance between $\mathbf{c}$ and $\mathbf{c}^{0}$ is defined by

$$
\sqrt{\sum_{i j k l}\left(c_{i j k l}-c_{i j k l}^{0}\right)^{2} \frac{1}{\sigma_{i j k l}^{2}}} .
$$

It should be straightforward to modify the available codes for finding the closest higher symmetric tensor in the Euclidean sense to include the standard deviations of the errors. In the following section, we see that this modification of the Euclidean metric produces substantially different results than the standard Euclidean approach.

\section{EXAMPLE}

Here, we find the tetragonal approximations of tensor 1 using the standard Euclidean metric and metric 15. Tetragonal tensors have the following form in their natural coordinates:

$$
\mathbf{S}=\left(\begin{array}{cccccc}
c_{11} & c_{12} & c_{13} & 0 & 0 & 0 \\
c_{12} & c_{11} & c_{13} & 0 & 0 & 0 \\
c_{13} & c_{13} & c_{33} & 0 & 0 & 0 \\
0 & 0 & 0 & 2 c_{44} & 0 & 0 \\
0 & 0 & 0 & 0 & 2 c_{44} & 0 \\
0 & 0 & 0 & 0 & 0 & 2 c_{66}
\end{array}\right)
$$

Using Mathematica ${ }^{\circledR}$ software and my method, I find the most probable tetragonal density-scaled stiffness tensor given by expressions 1 and 2 . The tensor in its natural coordinates is

$$
\mathbf{c}^{\text {Tetra }}=\left(\begin{array}{cccccc}
3.63221 & 2.28187 & 1.89512 & 0 & 0 & 0 \\
2.28187 & 3.63221 & 1.89512 & 0 & 0 & 0 \\
1.89512 & 1.89512 & 3.96287 & 0 & 0 & 0 \\
0 & 0 & 0 & 1.77481 & 0 & 0 \\
0 & 0 & 0 & 0 & 1.77481 & 0 \\
0 & 0 & 0 & 0 & 0 & 2.3094
\end{array}\right) \text {, }
$$

and the rotation to the measurement coordinate system is given by

$$
q=0.976916-0.160507 i+0.117906 j+0.0772653 k,
$$

which translates to the rotation matrix 


$$
\left(\begin{array}{rrr}
0.960256 & -0.188813 & 0.205565 \\
0.113114 & 0.936535 & 0.331824 \\
-0.255172 & -0.295384 & 0.945197
\end{array}\right) .
$$

We compare this result with the closest tetragonal tensor to $\mathbf{c}^{0}$ using the Euclidean metric on the space of tensors:

$$
\mathbf{c}_{\text {Euclid }}^{\text {terra }}=\left(\begin{array}{cccccc}
3.77886 & 2.2416 & 1.95904 & 0 & 0 & 0 \\
2.2416 & 3.77886 & 1.95904 & 0 & 0 & 0 \\
1.95904 & 1.95904 & 4.15289 & 0 & 0 & 0 \\
0 & 0 & 0 & 2.07723 & 0 & 0 \\
0 & 0 & 0 & 0 & 2.07723 & 0 \\
0 & 0 & 0 & 0 & 0 & 1.88492
\end{array}\right),
$$

with the rotation

$$
\left(\begin{array}{rrl}
-0.449253 & 0.728593 & -0.517034 \\
0.622961 & -0.159352 & -0.76585 \\
-0.640383 & -0.666153 & -0.382296
\end{array}\right) .
$$

Thus, two methods of finding the best approximation by a higher symmetric tensor result in two substantially different tensors.

\section{CONCLUSION}

The central point of this paper is to include knowledge of error distribution to establish the best approximation of the measured tensor by a higher symmetric tensor, considering the most likely symmetric tensor. In the case of normally distributed errors, this approximation is equivalent to the closest higher symmetric tensor in the modified Euclidean metric given by expression 15, where the standard deviations are included in the metric.

Two examples of error distribution have been presented. In the case of Gaussian distribution, there is a unique solution to the problem of finding the most likely tensor. In the case of box-cart error distribution, there are many possible solutions. To reduce this nonuniqueness, one can introduce additional constraints on the resulting tensors. A possible constraint could be the shortest distance using one of the more traditional metrics.

The most likely higher symmetric tensor can differ substantially from the closest higher symmetric tensor. The presented method results in the most likely tensor of the higher symmetry, so it is more suitable for situations in which we know the error distribution for the measurements of the elasticity tensors.

\section{ACKNOWLEDGMENTS}

I would like to thank the participants of the 13th International Workshop on Seismic Anisotropy (IWSA) for a fruitful discussion. In particular, comments of Paul Fowler were very helpful for the current form of the paper. Also, the use of quaternions was motivated by M. Kochetov and M. A. Slawinski (2009).

\section{REFERENCES}

Altmann, S. L., 1986, Rotations, quaternions, and double groups: Dover Publ. Inc.

Arsigny V., P. Fillard, X. Pennec, and N. Ayache, 2005, Fast and simple calculus on tensors in the log-Euclidean framework: Proceedings of the 8th International Conference on Medical Image Computing and ComputerAssisted Intervention, 115-122.

Arts, R., 1993, A study of general anisotropic elasticity in rocks by wave propagation: Theoretical and experimental aspects: Ph.D. thesis, University Pierre et Marie Curie.

Arts, R., K. Helbig, and P. Rasolofosaon, 1991, General anisotropic elastic tensor in rocks: Approximation, invariants, and particular directions: 61st Annual International Meeting, SEG, Expanded Abstracts, 1534-1537.

Bóna, A., I. Bucataru, and M. A. Slawinski, 2004, Material symmetries of elasticity tensors: Quarterly Journal of Mechanics and Applied Mathematics, 57, 583-598.

, 2007, Coordinate-free characterization of elasticity tensor: Journal of Elasticity, 87, 109-132.

Chadwick, P., M. Vianello, and S. Cowin, 2001, A new proof that the number of linear elastic symmetries is eight: Journal of the Mechanics and Physics of Solids, 49, 2471-2492.

Dellinger, J., 2005, Computing the optimal transversely isotropic approximation of a general elastic tensor: Geophysics, 70, no. 5, I1-I10.

Fedorov, F., 1968, Theory of elastic waves in crystals: Plenum Press

Forte, S., and M. Vianello, 1996, Symmetry classes for elasticity tensors: Journal of Elasticity, 43, 81-108.

Gazis, D. C., I. Tadjbakhsh, and R. A. Toupin, 1963, The elastic tensor of given symmetry nearest to an anisotropic elastic tensor: Acta Crystallographica, 16, 917-922.

Hamilton W., 1844, On a new species of imaginary quantities connected with a theory of quaternions: Proceedings of the Royal Irish Academy, 2, 424-434.

Kochetov, M., and M. A. Slawinski, 2009, On obtaining effective transversely isotropic elasticity tensors: Journal of Elasticity, 94, 1-13.

Mehrabadi, M., and S. Cowin, 1990, Eigentensors of linear anisotropic elastic materials: Quarterly Journal of Mechanics and Applied Mathematics, 43, 15-41.

Moakher, M., 2006, On the averaging of symmetric positive-definite tensors: Journal of Elasticity, 82, 273-296.

Moakher, M., and A. Norris, 2006, The closest elastic tensor of arbitrary symmetry to an elasticity tensor of lower symmetry: Journal of Elasticity, $\mathbf{8 5}, 215-263$.

Norris, A., 2006, Elastic moduli approximation of higher symmetry for the acoustical properties of an anisotropic material: Journal of the Acoustical Society of America, 119, 2114-2121.

Ting, T., 2003, Generalized Cowin-Mehrabadi theorems and a direct proof that the number of linear elastic symmetries is eight: International Journal of Solids and Structures, 40, 7129-7142. 